\title{
关于 $L_{p}(\phi)$ 估 计 \\ 丁夏畦罗佩珠 \\ (中国科学院数学研究所)
}

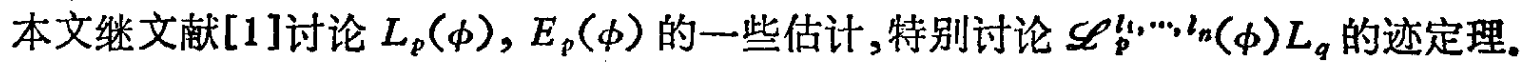

\section{1. 空间 $L_{p}\left(z^{\lambda} \phi_{1}\right), E_{p}\left(z^{\lambda} \phi_{1}\right)$}

定义 设 $\phi_{1}(z)=\sum_{m=0}^{\infty} a_{m} z^{m}$ 为一具非负系数的整函数, 如果定义在 $E_{n}$ 中某域 $Q(\Omega$ 可 为有界域或无界域)中的可测函数 $u(x)$ ，存在一正数 $\alpha$, 使

$$
\int_{\Omega} \phi\left(\alpha|u|^{p}\right) d x<\infty,
$$

则称 $u \in L_{p}(\phi, \Omega)$, 如果对所有的实 $\alpha>0,(1)$ 式均成立, 则称 $u \in E_{p}(\phi, \Omega), \phi=z^{2} \varphi_{1}(z)$ ， 若 $\lambda=1$, 则该空间就是我们过去所研究过的,我们有

定理 1 如果对应于两正系数整函数 $\phi_{1}(z)$ 与 $\phi_{2}(z)$ 的牛顿多边形 ${ }^{[2]} K_{\phi_{1}} \subseteq K_{\phi_{2}}$, 则

$$
L_{p}\left(z^{\lambda+\rho_{1}+\varepsilon} \phi_{2}, \Omega\right) \subseteq L_{p}\left(z^{\lambda} \phi_{1}, \Omega\right),
$$

$\rho_{1}$ 是 $\phi_{1}$ 的级, 其中 $\Omega$ 为有界域, 如果 $\phi_{2}$ 具全正规增长, 则必 $L_{p}\left(z^{\lambda} \phi_{2}, Q\right) \subset L_{p}\left(z^{\lambda} \phi_{1}, \Omega\right)$.

如果我们应用文献 [3] 中第四部第一章习题 57, 则可将上面的 $\rho_{1}+\varepsilon$ 改为 $\rho_{1} / 2$.

引理 对任何 Orlicz 空间 $L_{\Phi}$, 我们有

$$
\left\|\int_{Q} f(x, y) d y\right\|_{L_{\Phi}} \leqslant \int_{Q}\|f(x, y)\|_{L_{\Phi}} d y,
$$

此处 \|\|$_{L_{\Phi}}$ 系指 Luxemberg 范数, 即我们上面在 $L_{p}(\phi)$ 中所用者.

\section{2. 空间 $\mathscr{L}_{p}^{l_{p}}, \cdots, I_{n}\left(\phi ; E_{n-1}\right) L_{q}\left(E_{1}^{+}\right)$}

我们引人如下的范数

$$
\sum_{i=1}^{n}\left\{\int_{0}^{\infty} \frac{\left\|\Delta_{i, h}^{2} D_{i}^{i} f\right\|_{L_{p}\left(\phi ; E_{n}\right)}^{q}}{h^{1+q \lambda_{i}}} d h\right\}^{\frac{1}{q}}<\infty,
$$

其中 $\bar{l}_{i}$ 为小于 $l_{i}$ 的最大整数, 而 $l_{i}=\bar{l}_{i}+\lambda_{i}, 0<\lambda_{i} \leqslant 1$. 我们把光滑函数在上述范数下的

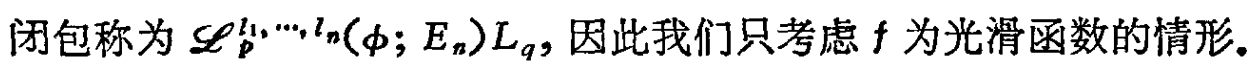

对于一般抛物组的 $L_{p}$ 估计, 文献 [4] 中已经系统研究了, 我们现仅考虑

$$
\left\{\begin{array}{l}
\frac{\partial u}{\partial t}=\Delta u, \\
u(x, 0)=u_{0}(x),
\end{array}\right.
$$

其中 $u_{0}(x) \in \mathscr{L}_{p}^{2 l}, \cdots, 2 l\left(\phi ; E_{n}\right) L_{q}$, 我们有

定理 2 对于初值问题 (2) 式的解 $u(x, t)$ 有

本文 1978 年 7 月 18 日收到。 


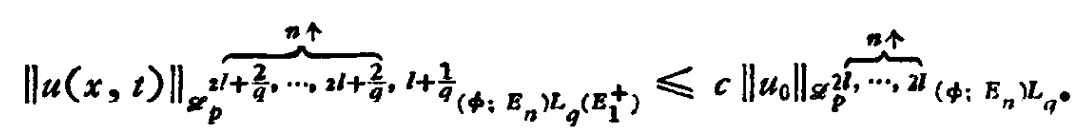

我们有

引理 $1\left\|\Delta_{i, h}^{m} f\right\|_{L_{p}\left(\phi ; E_{n}\right)} \leqslant h^{r_{i}}\left\|\Delta_{i, h}^{m}-r_{i} D^{r_{i} f}\right\|_{\left.l_{p(\phi ;} ; E_{n}\right)}$

引理 2

$$
\left(\int_{0}^{\infty} \frac{\left\|\Delta_{i, h}^{m} D_{1} f\right\|^{q}}{h^{1+q l}} d h\right)^{1 / q} \leqslant \frac{c}{a}\left(\int_{0}^{\infty} \frac{\left\|\Delta_{i, h}^{m} f\right\|^{q}}{h^{1+q(l+\rho)}} d h\right)^{1 / q}+c a^{\frac{1}{\rho}}\left(\int_{0}^{\infty} \frac{\left\|\Delta_{l, \xi}^{k} D_{1} f\right\|^{q}}{h^{1+\frac{1}{\rho} q}} d \xi\right)^{1 / q},
$$

其中 $\rho>0, l>0,\|\cdot\|$ 表示 $\|\cdot\|_{L_{p}\left(\phi ; E_{n-1}\right) L_{q}\left(E_{1}^{+}\right)} \cdot$

引理 3 当 $k \neq l_{i}$ 时, 则有

$$
\left(\int_{0}^{\infty} \frac{\left\|\Delta_{i, h}^{k} f\right\|^{q}}{h^{1+q l_{i}}} d h\right)^{1 / q} \leqslant c\left(\int_{0}^{\infty} \frac{\left\|\Delta_{i, k}^{k}, f\right\|^{q}}{h^{1+q l_{i}}} d h\right)^{1 / q} .
$$

定理 3 范数 $\|f\|_{\left.\alpha_{p}^{l i(\phi ;} B_{n-1}\right) L_{q}\left(E_{1}^{+}\right)}$

与

$$
\left(\int_{0}^{\infty} \frac{\| \Delta_{i b h}^{m} D^{r_{i} f \|^{q}}}{h^{1+q\left(l_{i}-r_{i}\right)}} d h\right)^{1 / q}
$$

等价.

其中 $m>l_{i}-r_{i}>0$.

为了下面的需要, 我们还有

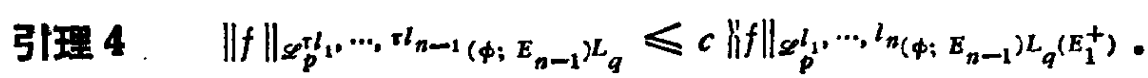

其中

$$
\tau=1-\frac{1}{q l_{n}}>0 .
$$

由此引理, 再运用数学归纳法, 就得到

定理 4 如果 $f \in \mathscr{L}_{p}^{l_{1}, \cdots, l_{n}}\left(\phi ; E_{n-1}\right) L_{q}\left(E_{1}^{+}\right)$, 则有

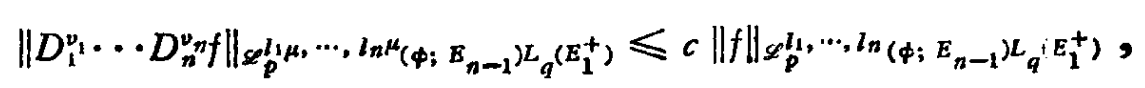

其中

$$
\mu=1-\sum_{j=1}^{n} \frac{v_{1}}{l_{i}}>0 .
$$

\section{3. 空间 $\mathscr{L}_{p, i k}^{l_{1}, \ldots, l_{n}}\left(\phi ; E_{n-1}\right) L_{q}\left(\psi ; E_{1}^{+}\right)$}

\section{在这一节, 我们将把上节的结果再作一些推广, 首先定义范数}

$$
\begin{aligned}
& \|f\|_{\alpha_{p, k}^{l_{i}}\left(\phi ; E_{n-1}\right) L_{q}\left(B_{1}^{+}\right)}=\left(\int_{0}^{\infty} \frac{d h}{h^{1+k l_{1}}}\left\|\frac{\Delta_{i, h f}^{n}}{h^{l_{i}}}\right\|^{q}\right)^{1 / q},
\end{aligned}
$$

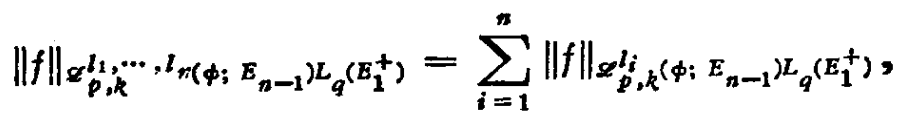

$$
\begin{aligned}
& \|f\|_{\alpha_{p, k}^{l i}\left(\phi ; E_{n-1}\right) L_{q}\left(\psi ; E_{1}^{+}\right)}=\inf \left\{\left(\frac{1}{\alpha}\right)^{1 / q}, \int_{0}^{\infty} \frac{d h}{h^{1+k l}} \psi\left(\alpha\left\|\frac{\| \Delta_{i, h}^{m} f}{h^{l} f}\right\|^{q}\right) \leqslant 1\right\},
\end{aligned}
$$




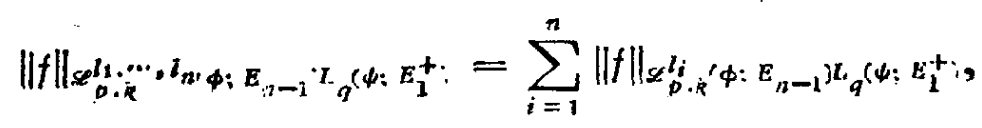

光滑函数在上述诸范数下之闭梢, 分别构成记于范数下标之空间.

由前面的定理,我们可以推出

定理 5 设 $f \in \mathscr{L}_{p, k, k}^{l, \cdots, i_{n}}\left(\phi ; E_{n-1}\right) L_{q}\left(E_{1}^{+}\right)$,

则

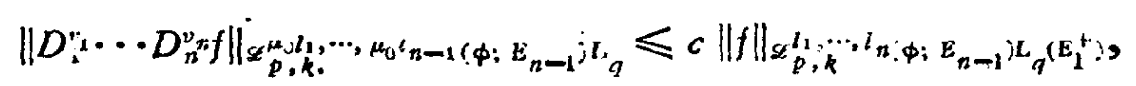

其中

$$
\mu_{\nu}=1-\sum_{i=1}^{\infty} \frac{\nu_{j}}{l_{j}}, \quad \mu_{1}=\frac{1}{l_{n}}, \quad \mu=u_{j}-\frac{\mu_{i}}{q}>0, \quad k_{1}=\left(k-\mu_{1}\right) \mu_{j}^{-1} .
$$

证 我们只须注意到

$$
\begin{aligned}
& \|f\|_{\alpha_{p, k}^{l}\left(\phi ; E_{n-1}, L_{q}\left(E_{1}^{+}\right)\right.}=\|f\|_{\mathcal{S}_{p, k}^{l} l_{i}^{\left(1+\frac{k}{4}\right)}\left(\phi ; E_{n-1}\right) L_{q}\left(E_{1}^{+}\right),},
\end{aligned}
$$

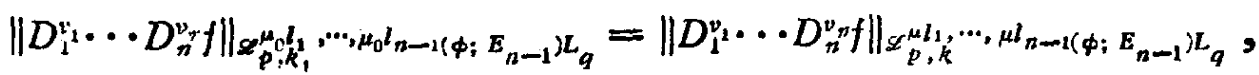

再应用 (7) 式, 就能推出定理.

根据定理 5 , 我们不难推出

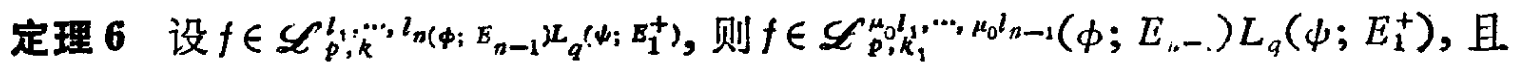

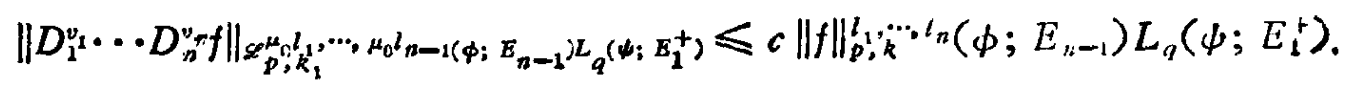

其中

$$
\psi=\sum_{m=1}^{\infty} b_{m} \mathrm{Z}^{m}
$$

为正系数整函数.

同样,对热传导方程的初值问题

$$
\left\{\begin{array}{l}
\frac{\partial u}{\partial t}-\Delta u=0, \\
u(x, 0)=u_{u}(x),
\end{array}\right.
$$

$u_{0} \in \mathscr{L}_{p, k}^{2 l, \cdots, 2 l}\left(\phi ; E_{n-1}\right) L_{q}\left(\psi ; E_{1}^{+}\right)$, 我们有

定理 7 对于上述初值问题的解 $u(x, t)$ ，我们有估计

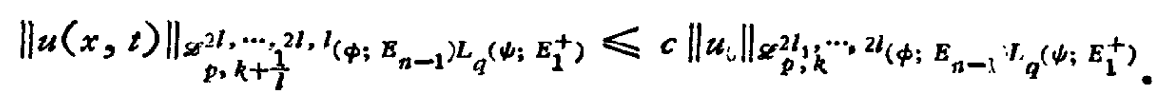

证只须注意到

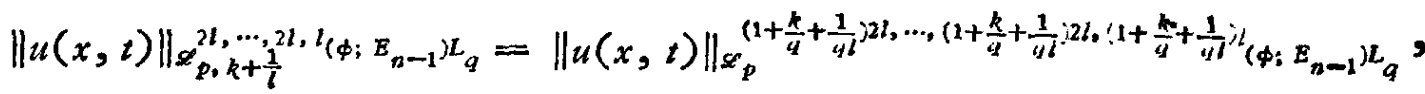

$$
\begin{aligned}
& \left\|u_{0}(x)\right\|_{\left.\right|_{p, k} ^{2 l}, \ldots, 2 l}\left(\phi ; E_{n-1}\right) L_{q}=\left\|u_{0}(x)\right\|_{x_{p}}\left(1+\frac{k}{q}\right) 2 l, \ldots, i+\frac{k}{q} 2 l_{\left(\phi ; E_{n-1}\right)} L_{q},
\end{aligned}
$$

从定理 2 即可得出本定理. 


\title{
$\Rightarrow$ 考文献
}

[1] 丁夏畔, 科学通报, 24 (1979), 6: 244-246.

[2] Valiron, G., Lecturer on the General Theory of Integral Functions, Chelsea Publishing Company, New York, 1925, 1-208.

[ 3 ] Полиа Г. и Сеге L.. Зидциа и теоремы из Анализа, Часть вторая, I осударственное издательство 'ехнико-теорегиче'кон литературы, Москва, 1956, 1-432.

[4] Wang Ruu-hwai, Scientia Sinica, XIV (1965), 9: 1373-1376.

\section{ON $L_{p}(\phi)$ ESTIMATES}

\section{Ding Xia-xi (丁夏畦) and Lo Pei-chu (罗佩珠)}

\begin{abstract}
In this paper are discussed the trace theorems of the spaces $\mathscr{L}_{p} \boldsymbol{l}_{1} \cdots \boldsymbol{l}_{n} \boldsymbol{L}_{p}(\phi) \mid \mathrm{g}$ which generalized the spaces $\mathscr{L}_{p}, \cdots, l_{n}(Q)$ irvestigated by B. A. Солоников. Then they have been applied to the a priori estimates of parabolic equations and some of the results have been generalized.
\end{abstract}

